\title{
Equivalence of Semantics in Argumentation
}

\author{
Leila Amgoud, Vivien Beuselinck \\ CNRS - UPS - IRIT, France \\ amgoud@irit.fr, vivien@beuselinck.fr
}

\begin{abstract}
A large number of evaluation methods, called semantics, have been proposed in the literature for assessing strength of arguments. This paper investigates their equivalence. It argues that for being equivalent, two semantics should have compatible evaluations of both individual arguments and pairs of arguments. The first requirement ensures that the two semantics judge an argument in the same way, while the second states that they provide the same ranking of arguments. We show that the two requirements are completely independent.

The paper introduces three novel relations between semantics based on their rankings of arguments: weak equivalence, strong equivalence and refinement. They state respectively that two semantics do not disagree on their strict rankings; the rankings of the semantics coincide; one semantics agrees with the strict comparisons of the second and it may break some of its ties. We investigate the properties of the three relations and their links with existing principles of semantics, and study the nature of relations between most of the existing semantics. The results show that the main extensions semantics are pairwise weakly equivalent. The gradual semantics we considered are pairwise incompatible, however some pairs are strongly equivalent in case of flat graphs including Maxbased (Mbs) and Euler-based (EMbs), for which we provide full characterizations in terms respectively of Fibonacci numbers and the numbers of an exponential series. Furthermore, we show that both semantics (Mbs, EMbs) refine the grounded semantics, and are weakly equivalent with the other extension semantics. We show also that in case of flat graphs, the two gradual semantics Trust-based and Iterative Schema characterize the grounded semantics, making thus bridges between gradual semantics and extension semantics. Finally, the other gradual semantics are incompatible with extension semantics.
\end{abstract}

\section{Introduction}

Argumentation is a reasoning approach based on the justification of claims by arguments. It has been used for solving different problems including inconsistency handling (Besnard and Hunter 2001; Amgoud and Cayrol 2002), decision making (Amgoud and Prade 2009; Zhong et al. 2019), casebased reasoning (Cyras, Satoh, and Toni 2016; Zheng, Grossi, and Verheij 2020), and negotiation (Dimopoulos, Mailly, and Moraitis 2019). See (Simari and Rahwan 2009) for more applications.
An argumentation-based system consists of a (flat or weighted) graph and an evaluation method, called a semantics. The nodes of the graph are arguments and its edges represent attacks between them. The graph is weighted when arguments are assigned basic weights and flat otherwise. The semantics is a formal method for evaluating the strength of every argument in the graph.

A great number of semantics have been proposed in the literature. They can roughly be classified into two families: extension-based and gradual. Initiated in (Dung 1995), the former look for sets of arguments (called extensions) that can be jointly accepted. Then, a dialectical status or strength is assigned to each argument according to its membership in the identified extensions. Introduced in (Cayrol and Lagasquie-Schiex 2005), gradual semantics focus on individual arguments, and ascribe to each of them a value taken from an ordered scale representing its strength.

Comparing existing semantics has been a hot topic in recent years. A dominant approach consists in identifying properties (called principles) that semantics may satisfy, then analysing every semantics against them. (Baroni and Giacomin 2007) proposed several principles that (van der Torre and Vesic 2017) used for comparing all the existing extension semantics. (Amgoud and Ben-Naim 2016) introduced another list of principles and used it for comparing some existing gradual/extension semantics. While these studies revealed conceptual differences between the analysed semantics, the messages they convey are not clear. Namely, the following questions remain unanswered:

- Are semantics satisfying the same principles similar (i.e. they provide the same evaluation of arguments)?

- Are semantics following different principles incompatible (i.e. they may provide contradictory evaluations)?

More generally, when are two semantics similar? We argue that to be similar, two semantics should agree not only on the evaluation of every individual argument but also on the ranking of arguments wrt their strengths. The first agreement depends on the principles followed by the two semantics. For instance, if both semantics satisfy the maximality principle from (Amgoud and Ben-Naim 2016), then they would assign the highest strength to a non-attacked argument. However, satisfying the same principles does not 
guarantee agreement on the ranking of arguments. This is particularly the case for the two gradual semantics Mbs (Amgoud et al. 2017) and EMbs (Amgoud and Doder 2019), which satisfy the same set of principles but may provide contradictory rankings of arguments when graphs are weighted. They are thus not similar and decision systems using them may make opposite recommendations. Consider for instance the case of a committee which recruits a new researcher, and assume there are two candidates Carla and Paul who are supported respectively by the arguments $a$ and $b$. Assume also that Mbs declares $a$ as stronger than $b$ and EMbs proposes the opposite ranking. Note that according to the semantics that is considered, the committee may make different recruitments (Carla with Mbs and Paul with EMbs).

To sum up, the existing comparisons of semantics are incomplete as they focused only on the first requirement for semantics similarity. This paper completes them by investigating the second requirement on rankings. Its contributions are threefold: First, it introduces three novel relations between semantics based on their rankings of arguments: weak equivalence, strong equivalence and refinement. They state respectively that two semantics do not disagree on their strict rankings; the rankings of the semantics coincide; one semantics agrees with the strict comparisons of the second and may break some of its ties. Second, it investigates the properties of the three relations and their links with existing principles of semantics. Third, it studies the nature of relations between most of the existing semantics when dealing with flat or weighted graphs. The results show the following:

- The main extensions semantics (grounded, ideal, stable, preferred) are pairwise weakly equivalent.

- The gradual semantics we studied are pairwise incompatible in case of weighted graphs. However, some pairs are strongly equivalent when graphs are flat, namely the pair Iterative Schema (IS) (Gabbay and Rodrigues 2015) and Trust-based (TB) (da Costa Pereira, Tettamanzi, and Villata 2011), and the pair Mbs and EMbs for which we provide novel characterizations in terms respectively of the Fibonacci numbers and the numbers of an exponential series.

- In case of flat graphs, we show that the gradual semantics TB and IS are two alternative characterizations of the grounded semantics. Consequently, the three are pairwise strongly equivalent. Furthermore, both Mbs and EMbs refine the grounded semantics (thus TB and IS). They are thus more discriminating since they provide finer evaluations of arguments. Note that this is crucial in applications like decision making where the more discriminating a model, the more efficient it is. These results make the first bridges between the two families of semantics (extension-based and gradual). The four semantics (TB, IS, Mbs, EMbs) are weakly equivalent with ideal, stable and preferred semantics while the other gradual semantics are incompatible with extension semantics.

The paper is structured as follows: Section 2 recalls basic notions, Section 3 defines novel relations between semantics, Sections 4 and 5 compare rankings produced by exten- sion semantics and gradual semantics respectively, Section 6 compares extension semantics with gradual ones, Section 7 is devoted to related work, and the last section concludes.

\section{Background}

Let us introduce some useful notions for the rest of the paper.

Definition 1 (Weighting). A weighting on a set $X$ is a function from $X$ to $[0,1]$.

A preordering on a set of objects is a binary relation that is reflexive and transitive.

Definition 2 (Preordering). A preordering on a set $X$ is a binary relation $\succeq$ on $X$ such that:

- For any $x \in X, x \succeq x$

(Reflexivity)

- For all $x, y, z \in X$, if $x \succeq y$ and $y \succeq z$, then $x \succeq z$

(Transitivity)

A preordering $\succeq$ is total iff for all $x, y \in X, x \succeq y$ or $y \succeq x$.

The notation $x \succ y$ is a shortcut for $x \succeq y$ and $y \nsucceq x$ and $x \approx y$ is a shortcut for $x \succeq y$ and $y \succeq x$. Throughout the paper, the relation $\succ$ is the strict version of $\succeq$, i.e. $\succ=$ $\left\{(x, y) \in X^{2} \mid x \succ y\right\}$, and $\approx$ is the equivalence relation, i.e. $\approx=\left\{(x, y) \in X^{2} \mid x \succeq y\right.$ and $\left.y \succeq x\right\}$.

Property 1. If a binary relation $\succeq$ is a total preordering, then $\succeq=\succ \cup \approx$.

Throughout the paper, we consider argumentation graphs whose nodes are arguments, each of which has a basic weight representing an aggregation of votes given by users (Leite and Martins 2011), or a certainty degree of the argument's premises (Benferhat, Dubois, and Prade 1993), or a trustworthiness degree of its source (da Costa Pereira, Tettamanzi, and Villata 2011), etc. Edges represent attacks (i.e. conflicts) between arguments. For the sake of simplicity, basic weights are elements of the unit interval $[0,1]$. The greater the value, the stronger the argument. We denote by Args the universe of all arguments.

Definition 3 (AG). An argumentation graph $(A G)$ is a tuple $\mathrm{G}=\langle\mathcal{A}, \sigma, \mathcal{R}\rangle$, with $\mathcal{A} \subseteq{ }_{f} \operatorname{Args}^{1}, \mathcal{R} \subseteq \mathcal{A} \times \mathcal{A}$ is an attack relation, $\sigma$ is a weighting on $\mathcal{A}$. G is flat if $\sigma \equiv 1^{2}$ and weighted otherwise. Let $\mathrm{AG}$ denote the set of all argumentation graphs that can be built from Args.

Let us now define the notion of semantics. It is a function that assigns a value from a given ordered scale to each argument. The value represents the strength of the argument. The greater it is, the stronger the argument. Different scales can be used, but for simplicity we use the unit interval $[0,1]$.

Definition 4 (Semantics). A semantics is a function $\mathbf{S}$ assigning to any $\mathrm{G}=\langle\mathcal{A}, \sigma, \mathcal{R}\rangle \in \mathrm{AG}$ a weighting $\operatorname{Deg}_{\mathrm{G}}^{\mathrm{S}}$ on $\mathcal{A}$. For any $a \in \mathcal{A}, \operatorname{Deg}_{\mathrm{G}}^{\mathbf{S}}(a)$ represents the strength of $a$. Let Sem denote the set of all possible semantics.

We introduce next some useful notations.

Notations: Let $\mathrm{G}=\langle\mathcal{A}, \sigma, \mathcal{R}\rangle \in \mathrm{AG}$ and $a \in \mathcal{A}$. $\operatorname{Att}_{\mathrm{G}}(a)$ denotes the set $\{b \in \mathcal{A} \mid(b, a) \in \mathcal{R}\}$ of direct attackers of

\footnotetext{
${ }^{1} \mathcal{A} \subseteq$ Args means $\mathcal{A}$ is a finite subset of Args.

${ }^{2} \sigma \equiv 1$ means $\forall a \in \mathcal{A}, \sigma(a)=1$.
} 
$a$ in $\mathrm{G}$. When $\mathrm{G}$ is clear from the context, we write $\operatorname{Att}(a)$ for short. Let $\mathrm{G}^{\prime}=\left\langle\mathcal{A}^{\prime}, \sigma^{\prime}, \mathcal{R}^{\prime}\right\rangle \in \mathrm{AG}$ such that $\mathcal{A} \cap \mathcal{A}^{\prime}=\emptyset$. $\mathrm{G} \oplus \mathrm{G}^{\prime}=\left\langle\mathcal{A} \cup \mathcal{A}^{\prime}, \sigma^{\prime \prime}, \mathcal{R} \cup \mathcal{R}^{\prime}\right\rangle \in \mathrm{AG}$ such that $\forall x \in \mathcal{A}$ (resp. $\left.x \in \mathcal{A}^{\prime}\right), \sigma^{\prime \prime}(x)=\sigma(x)\left(\right.$ resp. $\left.\sigma^{\prime \prime}(x)=\sigma^{\prime}(x)\right)$.

We recall below the list of principles proposed in (Amgoud et al. 2017) for semantics $\mathbf{S} \in$ Sem.

Anonymity. $\forall \mathrm{G}=\langle\mathcal{A}, \sigma, \mathcal{R}\rangle, \mathrm{G}^{\prime}=\left\langle\mathcal{A}^{\prime}, \sigma^{\prime}, \mathcal{R}^{\prime}\right\rangle \in \mathrm{AG}$, for any isomorphism $f$ from $\mathrm{G}$ to $\mathrm{G}^{\prime}, \forall a \in \mathcal{A}, \operatorname{Deg}_{\mathrm{G}} \mathbf{S}(a)=$ $\operatorname{Deg}_{G^{\prime}}^{\mathbf{S}}(f(a))$.

Independence. $\forall \mathrm{G}=\langle\mathcal{A}, \sigma, \mathcal{R}\rangle, \mathrm{G}^{\prime}=\left\langle\mathcal{A}^{\prime}, \sigma^{\prime}, \mathcal{R}^{\prime}\right\rangle \in \mathrm{AG}$ such that $\mathcal{A} \cap \mathcal{A}^{\prime}=\emptyset, \forall a \in \mathcal{A}, \operatorname{Deg}_{\mathrm{G}}^{\mathbf{S}}(a)=\operatorname{Deg}_{\mathrm{G} \otimes \mathrm{G}^{\prime}}^{\mathbf{S}}(a)$.

Directionality. $\forall \mathrm{G}=\langle\mathcal{A}, \sigma, \mathcal{R}\rangle, \mathrm{G}^{\prime}=\left\langle\mathcal{A}, \sigma, \mathcal{R}^{\prime}\right\rangle \in \mathrm{AG}$ such that $a, b \in \mathcal{A}, \mathcal{R}^{\prime}=\mathcal{R} \cup\{(a, b)\}$ it holds that: $\forall x \in \mathcal{A}$, if there is no path from $\mathrm{b}$ to $\mathrm{x}$, then $\operatorname{Deg}_{\mathrm{G}}^{\mathrm{S}}(x)=\operatorname{Deg}_{\mathrm{G}^{\prime}} \mathbf{S}(x)$.

Neutrality. $\forall \mathrm{G}=\langle\mathcal{A}, \sigma, \mathcal{R}\rangle \in \mathrm{AG}, \forall a, b \in \mathcal{A}$, if $\sigma(a)=$ $\sigma(b), \operatorname{Att}_{G}(b)=\operatorname{Att}_{G}(a) \cup\{x\}$ with $x \in \mathcal{A} \backslash \operatorname{Att}_{G}(a)$ and $\operatorname{Deg}_{G}^{\mathbf{S}}(x)=0$, then $\operatorname{Deg}_{G}^{\mathbf{S}}(a)=\operatorname{Deg}_{G}^{\mathbf{S}}(b)$.

Equivalence. $\forall \mathrm{G}=\langle\mathcal{A}, \sigma, \mathcal{R}\rangle \in \mathrm{AG}, \forall a, b \in \mathcal{A}$, if $\sigma(a)=$ $\sigma(b)$ and there exists a bijective function $\mathrm{f}_{\text {from } \operatorname{Att}_{\mathrm{G}}(a)}$ to $\operatorname{Att}_{G}(b)$ s.t. $\forall x \in \operatorname{Att}_{G}(a), \operatorname{Deg}_{G} \mathbf{S}(x)=\operatorname{Deg}_{G} \mathbf{S}(f(x))$, then $\operatorname{Deg}_{G}^{\mathbf{S}}(a)=\operatorname{Deg}_{G}^{\mathbf{S}}(b)$.

Maximality. $\forall \mathrm{G}=\langle\mathcal{A}, \sigma, \mathcal{R}\rangle \in \mathrm{AG}, \forall a \in \mathcal{A}$, if $\operatorname{Att}_{\mathrm{G}}(a)=$ $\emptyset$, then $\operatorname{Deg}_{G}^{\mathbf{S}}(a)=\sigma(a)$.

Counting. $\forall \mathrm{G}=\langle\mathcal{A}, \sigma, \mathcal{R}\rangle \in \mathrm{AG}, \forall a, b \in \mathcal{A}$, if $\left.\operatorname{Deg}_{\mathrm{G}}^{\mathbf{S}}(a)\right\rangle$ 0 and $\operatorname{Att}_{G}(b)=\operatorname{Att}_{G}(a) \cup\{x\}$ with $x \in \mathcal{A} \backslash \operatorname{Att}_{G}(a)$ and $\operatorname{Deg}_{G}^{\mathbf{S}}(x)>0$, then $\operatorname{Deg}_{G}^{\mathbf{S}}(a)>\operatorname{Deg}_{G}^{\mathbf{S}}(b)$.

Weakening. $\forall \mathrm{G}=\langle\mathcal{A}, \sigma, \mathcal{R}\rangle \in \mathrm{AG}, \forall a \in \mathcal{A}$, if $\exists b \in \operatorname{Att}_{\mathrm{G}}(a)$ s.t. $\operatorname{Deg}_{G}^{\mathbf{S}}(b)>0$ and $\sigma(a)>0$, then $\operatorname{Deg}_{G}^{\mathbf{S}}(a)<\sigma(a)$.

Weakening Soundness. $\forall \mathrm{G}=\langle\mathcal{A}, \sigma, \mathcal{R}\rangle \in \mathrm{AG}$, if $\operatorname{Deg}_{G} \mathbf{S}(a)<$ $\sigma(a)$, then $\exists b \in \operatorname{Att}_{G}(a)$ s.t $\operatorname{Deg}_{G}^{\mathbf{S}}(b)>0$.

Reinforcement. $\forall \mathrm{G}=\langle\mathcal{A}, \sigma, \mathcal{R}\rangle \in \mathrm{AG}, \forall a, b \in \mathcal{A}$, if i) $\sigma(a)=\sigma(b)$, ii) $\operatorname{Deg}_{G}^{\mathbf{S}}(a)>0$ or $\operatorname{Deg}_{G}^{\mathbf{S}}(b)>0$, iii) $\operatorname{Att}_{\mathrm{G}}(a) \backslash \operatorname{Att}_{\mathrm{G}}(b)=\{x\}$, iv) $\operatorname{Att}_{\mathrm{G}}(b) \backslash \operatorname{Att}_{\mathrm{G}}(a)=\{y\}$, v) $\operatorname{Deg}_{G}^{\mathbf{S}}(y)>\operatorname{Deg}_{G}^{\mathbf{S}}(x)>0$, then $\operatorname{Deg}_{G}^{\mathbf{S}}(a)>\operatorname{Deg}_{G}^{\mathbf{S}}(b)$.

Resilience. $\forall \mathrm{G}=\langle\mathcal{A}, \sigma, \mathcal{R}\rangle \in \mathrm{AG}, \forall a \in \mathcal{A}$, if $\sigma(a)>0$ then $\operatorname{Deg}_{G}^{\mathbf{S}}(a)>0$.

Proportionality. $\forall \mathrm{G}=\langle\mathcal{A}, \sigma, \mathcal{R}\rangle \in \mathrm{AG}, \forall a, b \in \mathcal{A}$, if $\sigma(a)>\sigma(b)$ and $\operatorname{Att}_{G}(a)=\operatorname{Att}_{G}(b)$, then $\operatorname{Deg}_{G} \mathbf{S}_{(a)}>$ $\operatorname{Deg}_{G}^{\mathbf{S}}(b)$.

Monotony. $\forall \mathrm{G}=\langle\mathcal{A}, \sigma, \mathcal{R}\rangle \in \mathrm{AG}, \forall a, b \in \mathcal{A}$, if $\sigma(a)=$ $\sigma(b)$ and $\operatorname{Att}(a) \subset \operatorname{Att}(b)$, then $\operatorname{Deg}_{G}^{\mathbf{S}}(a) \geq \operatorname{Deg}_{G}^{\mathbf{S}}(b)$.

Quality Precedence. $\forall \mathrm{G}=\langle\mathcal{A}, \sigma, \mathcal{R}\rangle \in \mathrm{AG}, \forall a, b \in \mathcal{A}$, if i) $\operatorname{Deg}_{G}^{\mathbf{S}}(a)>0$ and ii) $\exists y \in \operatorname{Att}_{G}(b)$ s.t. $\forall x \in \operatorname{Att}_{G}(a)$, $\operatorname{Deg}_{G}^{\mathbf{S}}(y)>\operatorname{Deg}_{G}^{\mathbf{S}}(x)$, then $\operatorname{Deg}_{G}^{\mathbf{S}}(a)>\operatorname{Deg}_{G}^{\mathbf{S}}(b)$.

Cardinality Precedence. $\forall \mathrm{G}=\langle\mathcal{A}, \sigma, \mathcal{R}\rangle \in \mathrm{AG}, \forall a, b \in$ $\mathcal{A}$, if i) $\sigma(a)=\sigma(b)$ ii) $\operatorname{Deg}_{G}^{\mathbf{S}}(b)>0$, and iii) $\mid\left\{x \in \operatorname{Att}_{G}(a)\right.$ s.t. $\left.\operatorname{Deg}_{G} \mathbf{S}_{(x)}>0\right\}|>|\{y \in$ $\operatorname{Att}_{G}(b)$ s.t. $\left.\operatorname{Deg}_{G}^{\mathbf{S}}(y)>0\right\} \mid$, then $\operatorname{Deg}_{G}^{\mathbf{S}}(a)<\operatorname{Deg}_{G}^{\mathbf{S}}(b)$.

Compensation. $\exists \mathrm{G}=\langle\mathcal{A}, \sigma, \mathcal{R}\rangle \in \mathrm{AG}$ s.t for two arguments $a, b \in \mathcal{A}$, i) $\sigma(a)=\sigma(b)$ ii) $\operatorname{Deg}_{G}^{\mathbf{S}}(a)>0$, iii) $\mid\{x \in$ $\left.\operatorname{Att}_{\mathrm{G}}(a) \mid \operatorname{Deg}_{\mathrm{G}} \mathbf{S}(x)>0\right\}|>|\left\{y \in \operatorname{Att}_{\mathrm{G}}(b) \mid \operatorname{Deg}_{\mathrm{G}} \mathbf{S}(y)>\right.$ $0\} \mid$, iv) $\exists y \in \operatorname{Att}_{G}(b)$ s.t. $\forall x \in \operatorname{Att}_{G}(a), \operatorname{Deg}_{G}^{\mathbf{S}}(y)>$ $\operatorname{Deg}_{G}^{\mathbf{S}}(x)$ and $\operatorname{Deg}_{G}^{\mathbf{S}}(a)=\operatorname{Deg}_{G}^{\mathbf{S}}(b)$.

\section{Notions of Equivalence}

We have seen in the previous section that a semantics assigns a numerical value to each argument in an argumentation graph. Those values are used for defining a preference relation between arguments as follows.

Definition 5 (Ranking). Let $\mathbf{S} \in$ Sem and $\mathrm{G}=\langle\mathcal{A}, \sigma, \mathcal{R}\rangle \in$ AG. $A$ ranking induced from $\mathbf{S}$ is a binary relation $\succeq_{\mathbf{S}}^{\mathrm{G}}$ on $\mathcal{A}$ such that for all $a, b \in \mathcal{A}$,

$$
a \succeq_{\mathbf{S}}^{\mathbf{G}} b \text { iff } \operatorname{Deg}_{G}^{\mathbf{S}}(a) \geq \operatorname{Deg}_{G}^{\mathbf{S}}(b) .
$$

The notation $a \succeq_{\mathrm{S}}^{\mathrm{G}} b$ expresses that the argument $a$ is at least as strong as the argument $b$ in the graph $\mathrm{G}$ under the semantics $\mathbf{S}$. Hence, $a \succ_{\mathbf{S}}^{\mathrm{G}} b$ and $a \approx_{\mathbf{S}}^{\mathrm{G}} b$ state respectively that $a$ is stronger than $b$ and $a$ is as strong as $b$ under $\mathbf{S}$.

The relation $\succeq^{\mathbf{S}}$ is obviously a total preordering, i.e. it is reflexive, transitive and compares every pair of arguments.

Property 2. For any $\mathbf{S} \in \mathrm{Sem}$, for any $\mathrm{G} \in \mathrm{AG}$, the ranking $\succeq_{\mathrm{S}}^{\mathrm{G}}$ is a total preordering.

In this section, we study when two semantics $\mathbf{S}_{1}$ and $\mathbf{S}_{2}$ are equivalent by comparing the rankings they provide. We introduce three relations between two rankings. The first relation is that of refinement. A semantics refines (or is more discriminating than) another semantics when it agrees with its strict comparisons and may break some of its ties.

Definition 6 (Refinement). Let $\mathbf{S}_{1}, \mathbf{S}_{2} \in \mathrm{Sem}$. We say that $\mathbf{S}_{1}$ refines $\mathbf{S}_{2}$ iff $\forall \mathrm{G} \in \mathrm{AG}, \succ_{\mathbf{S}_{2}}^{\mathrm{G}} \subseteq \succ_{\mathbf{S}_{1}}^{\mathrm{G}}$.

Property 3. The following properties hold:

- Let $\mathbf{S}_{1}, \mathbf{S}_{2} \in$ Sem. If $\mathbf{S}_{1}$ refines $\mathbf{S}_{2}$, then $\forall \mathrm{G} \in \mathrm{AG}$, the inclusion $\approx_{\mathbf{S}_{1}}^{\mathrm{G}} \subseteq \approx_{\mathbf{S}_{2}}^{\mathrm{G}}$ holds.

- The refinement relation is transitive.

The second notion, weak equivalence, ensures the compatibility of two rankings. More precisely, two semantics are weakly equivalent if they never provide opposite strict rankings of arguments. They are said to be incompatible otherwise. This notion is more general than refinement because neither of the two semantics should refine the other.

Definition 7 (Weak Equivalence). Let $\mathbf{S}_{1}, \mathbf{S}_{2} \in$ Sem. We say that $\mathbf{S}_{1}$ and $\mathbf{S}_{2}$ are weakly equivalent iff $\forall \mathrm{G}=\langle\mathcal{A}, \sigma, \mathcal{R}\rangle \in$ $\mathrm{AG}, \nexists a, b \in \mathcal{A}$ s.t. $a \succ_{\mathbf{S}_{1}}^{\mathrm{G}} b$ and $b \succ_{\mathbf{S}_{2}}^{\mathrm{G}} a$. The two semantics are said to be incompatible otherwise.

Weak equivalence is a binary relation over the set of all possible semantics. It is reflexive but not transitive. Indeed, for three semantics $\mathbf{S}_{1}, \mathbf{S}_{2}, \mathbf{S}_{3}$, it is possible that $\mathbf{S}_{1}, \mathbf{S}_{2}$ (resp. $\mathbf{S}_{2}, \mathbf{S}_{3}$ ) are weakly equivalent but $\mathbf{S}_{1}, \mathbf{S}_{3}$ are not.

Property 4. The weak equivalence relation is not transitive.

It is worthy to notice that two weakly equivalent semantics may not provide exactly the same ranking of arguments. Consider for instance four arguments $a, b, c, d$ and two semantics $\mathbf{S}_{1}$ and $\mathbf{S}_{2}$. One may have: $a \succ_{\mathbf{S}_{1}} b, c \approx_{\mathbf{S}_{1}} d$ and $a \approx_{\mathbf{S}_{2}} b, c \succ_{\mathbf{S}_{2}} d$.

The third notion of strong equivalence ensures total agreement of two semantics regarding their rankings.

Definition 8 (Strong Equivalence). Let $\mathbf{S}_{1}, \mathbf{S}_{2} \in$ Sem. We say that $\mathbf{S}_{1}$ and $\mathbf{S}_{2}$ are strongly equivalent iff for any $\mathrm{G}=$ $\langle\mathcal{A}, \sigma, \mathcal{R}\rangle \in \mathrm{AG}$, the equality $\succeq_{\mathbf{S}_{1}}^{\mathrm{G}}=\succeq_{\mathbf{S}_{2}}^{\mathrm{G}}$ holds. 
Since rankings induced by semantics are total preorderings, then two strongly equivalent semantics agree both on strict rankings and on ties.

Property 5. The following properties hold:

- Two semantics $\mathbf{S}_{1}, \mathbf{S}_{2}$ are strongly equivalent iff $\succ_{\mathbf{S}_{1}}=$ $\succ_{\mathbf{S}_{2}}$ and $\approx_{\mathbf{S}_{1}}=\approx_{\mathbf{S}_{2}}$.

- Strong equivalence is transitive.

The following property summarizes the different links between the above three relations.

Proposition 1. Let $\mathbf{S}_{1}, \mathbf{S}_{2}, \mathbf{S}_{3} \in$ Sem.

1. $\mathbf{S}_{1}, \mathbf{S}_{2}$ are strongly equivalent iff they refine each other.

2. If $\mathbf{S}_{1}$ refines $\mathbf{S}_{2}$, then $\mathbf{S}_{1}$ and $\mathbf{S}_{2}$ are weakly equivalent.

3. If $\mathbf{S}_{1}$ and $\mathbf{S}_{2}$ are strongly equivalent, then they are weakly equivalent. The converse does not hold.

4. If $\mathbf{S}_{1}$ and $\mathbf{S}_{2}$ are strongly equivalent and $\mathbf{S}_{2}$ and $\mathbf{S}_{3}$ are weakly equivalent, then $\mathbf{S}_{1}$ and $\mathbf{S}_{3}$ are weakly equivalent.

5. If $\mathbf{S}_{1}$ refines $\mathbf{S}_{2}$ and $\mathbf{S}_{1}$ is strongly equivalent to $\mathbf{S}_{3}$, then $\mathbf{S}_{3}$ refines $\mathbf{S}_{2}$.

6. If $\mathbf{S}_{1}$ and $\mathbf{S}_{2}$ are strongly equivalent and $\mathbf{S}_{2}$ and $\mathbf{S}_{3}$ are incompatible, then $\mathbf{S}_{1}$ and $\mathbf{S}_{3}$ are incompatible.

7. If $\mathbf{S}_{1}$ refines $\mathbf{S}_{2}$ and $\mathbf{S}_{2}$ and $\mathbf{S}_{3}$ are incompatible, then $\mathbf{S}_{1}$ and $\mathbf{S}_{3}$ are incompatible.

We show next that the principles recalled in the previous section are necessary but not sufficient for the equivalence of two semantics. Indeed, two semantics may satisfy the same set of principles without necessarily being strongly or weakly equivalent. Conversely, two semantics may be strongly equivalent while they do not follow the same principles. Before introducing the formal result, let us first introduce two notations.

Notations: Let $\operatorname{Princ}(\mathbf{S})$ denote the subset of principles satisfied by a semantics $\mathbf{S} \in$ Sem, and by $I$ the set containing the following principles: Neutrality, Equivalence, Counting, Reinforcement, Proportionality, and Monotony.

Proposition 2. The following properties hold:

- Let $\mathbf{S}_{1}, \mathbf{S}_{2} \in$ Sem and $x \in$ I. If $\mathbf{S}_{1}$ satisfies $x$ and $\mathbf{S}_{2}$ violates $x$, then $\mathbf{S}_{1}$ and $\mathbf{S}_{2}$ are not strongly equivalent.

- Let $\mathbf{S}_{1}, \mathbf{S}_{2} \in$ Sem. If $\mathbf{S}_{1}$ satisfies Cardinality Precedence and $\mathbf{S}_{2}$ satisfies Quality Precedence, then $\mathbf{S}_{1}$ and $\mathbf{S}_{2}$ are incompatible.

- $\exists \mathbf{S}_{1}, \mathbf{S}_{2} \in$ Sem such that $\mathbf{S}_{1}, \mathbf{S}_{2}$ are strongly equivalent and $\operatorname{Princ}\left(\mathbf{S}_{1}\right) \neq \operatorname{Princ}\left(\mathbf{S}_{2}\right)$.

- $\exists \mathbf{S}_{1}, \mathbf{S}_{2} \in$ Sem such that $\operatorname{Princ}\left(\mathbf{S}_{1}\right)=\operatorname{Princ}\left(\mathbf{S}_{2}\right)$ and $\mathbf{S}_{1}, \mathbf{S}_{2}$ are incompatible.

The above results show that for being similar, it is not sufficient that two semantics evaluate in the same way every individual arguments. They should also provide the same ranking of arguments. Existing comparisons of semantics focused exclusively on the first criterion by relying on the principles followed by semantics. In the next section, we will compare semantics wrt their rankings.

\section{Comparison of Extension Semantics}

Initially proposed by (Dung 1995) for evaluating arguments of flat graphs (i.e. graphs of the form $\langle\mathcal{A}, \sigma \equiv 1, \mathcal{R}\rangle$ ), extension semantics compute sets of jointly acceptable arguments. They are based on three key concepts: conflictfreeness, defense and admissibility. Let $\mathcal{E} \subseteq \mathcal{A}$,

- $\mathcal{E}$ is conflict-free iff $\nexists a, b \in \mathcal{E}$ such that $(a, b) \in \mathcal{R}$.

- $\mathcal{E}$ defends $a \in \mathcal{A}$ iff $\forall b \in \mathcal{A}$, if $(b, a) \in \mathcal{R}$, then $\exists c \in \mathcal{E}$ such that $(c, b) \in \mathcal{R}$.

- $\mathcal{E}$ is an admissible set iff it is conflict-free and defends all its elements.

We recall below the standard extension semantics from (Dung 1995) and the ideal semantics from (Dung, Mancarella, and Toni 2007).

- $\mathcal{E}$ is a preferred extension iff it is a maximal (wrt set inclusion) admissible extension.

- $\mathcal{E}$ is a stable extension iff it is conflict-free and $\forall a \in \mathcal{A} \backslash \mathcal{E}$, $\exists b \in \mathcal{E}$ such that $(b, a) \in \mathcal{R}$.

- $\mathcal{E}$ is an ideal extension iff it is a maximal (wrt set inclusion) admissible set that is subset of every preferred extension.

- $\mathcal{E}$ is a grounded extension iff it is the least fixpoint of the characteristic function $\mathcal{F}: 2^{\mathcal{A}} \rightarrow 2^{\mathcal{A}}$ where for $S \subseteq \mathcal{A}$,

$$
\mathcal{F}(S)=\{a \in \mathcal{A} \mid S \text { defends } a\}
$$

Let $\operatorname{Gr}(G)$ denote the grounded extension of $G$.

It is worth recalling that an argumentation graph has a single grounded (resp. ideal) extension. However, it may have zero, one or several stable extensions. Every stable extension is a preferred one, but the converse is not necessarily true. Throughout the paper we refer by $i, g, s, p$ respectively to ideal, grounded, stable and preferred semantics.

Given a set of extensions of a flat graph $\langle\mathcal{A}, \sigma \equiv 1, \mathcal{R}\rangle$, the most common way to assign a strength to an argument $a \in \mathcal{A}$ (see (Baroni, Giacomin, and Guida 2005; Cayrol and Lagasquie-Schiex 2005;

Grossi and Modgil 2015)) is as follows:

- $a$ is sceptically accepted if it belongs to all extensions,

- $a$ is credulously accepted it belongs to some but not all extensions,

- $a$ is rejected if it does not belong to any extension.

Since we considered a numerical scale for argument strength, we transform the three-valued qualitative scale into a numerical one following the approach of (Amgoud and Ben-Naim 2016). The idea is to use the scale $T=\{1, \alpha, \beta, 0\}$, with $1>\alpha>\beta>0$, and to assign the value 1 to sceptically accepted arguments, the value $\alpha$ to credulously accepted ones, and to distinguish two kinds of rejected arguments: those that are not attacked by any extension and which got value $\beta$, and those that are attacked by at least one extension and that got the minimal value 0 . Note that a set of arguments $\mathcal{E}$ attacks an argument $a$ if it contains at least one argument $b$ which attacks $a$. 


\begin{tabular}{|c|c|c|}
\hline Semantics & Definition & Graphs \\
\hline$h$-Categorizer (hCat) (Besnard and Hunter 2001) & $\operatorname{Deg}_{G}^{h}(a)=\frac{1}{1+\sum_{b \mathcal{R} a} \operatorname{Deg}_{G}^{h}(b)}$ & Flat \\
\hline Compensation-based ( $\alpha$-BBS) (Amgoud et al. 2016) & $\operatorname{Deg}_{G}^{\alpha-\operatorname{BBS}}(a)=1+\left(\sum_{b \mathcal{R} a} \frac{1}{(s(b))^{\alpha}}\right)^{1 / \alpha}, \alpha \in(0,+\infty)$ & Flat \\
\hline Weighted $h$-Categorizer (Hbs) (Amgoud et al. 2017) & $\operatorname{Deg}_{G}^{\mathrm{Hbs}}(a)=\frac{\sigma(a)}{1+\sum_{b \mathcal{R} a} \operatorname{Deg}_{G}^{\mathrm{Hbs}}(b)}$ & Weighted \\
\hline Weighted Card-based (Cbs) (Amgoud et al. 2017) & $\begin{array}{l}\operatorname{Deg}_{\mathrm{G}}^{\mathrm{Cbs}}(a)=\frac{\sigma(a)}{\sum_{1+\left|\operatorname{AttF}_{\mathrm{G}}(a)\right|+\frac{b \in \operatorname{Att}_{\mathrm{G}}(a)}{\operatorname{\operatorname {Deg}_{\mathrm {G}}\mathrm {Ctg}(b)}}} \text { where }} \\
\operatorname{AttF}_{\mathrm{G}}(a)=\left\{b \in \operatorname{Att}_{\mathrm{G}}(a) \mid \sigma(b)>0\right\}\end{array}$ & Weighted \\
\hline Weighted Max-based (Mbs) (Amgoud et al. 2017) & $\operatorname{Deg}_{G}^{\operatorname{Mbs}}(a)=\frac{\sigma(a)}{1+\max _{b \mathcal{R} a} \operatorname{Deg}_{G}^{\operatorname{Mbs}(b)}}$ & Weighted \\
\hline Euler-Max-based (EMbs) (Amgoud and Doder 2019) & $\operatorname{Deg}_{\mathrm{G}}^{\mathrm{EMbs}}(a)=\sigma(a) \cdot e^{-\max _{b \mathcal{R} a} \operatorname{Deg}_{\mathrm{G}}^{\mathrm{E} M \mathrm{bss}}(b)}$ & Weighted \\
\hline $\begin{array}{l}\text { Trust-based (TB) } \\
\text { (da Costa Pereira, Tettamanzi, and Villata 2011) }\end{array}$ & $\begin{array}{l}\operatorname{Deg}_{G}^{\mathrm{IB}}(a)=\lim _{i \rightarrow+\infty} f_{i}(a), \text { where } \\
f_{i}(a)=\frac{1}{2} f_{i-1}(a)+\frac{1}{2} \min \left[w(a), 1-\max _{b \mathcal{R} a} f_{i-1}(b)\right]\end{array}$ & Weighted \\
\hline Iterative Schema (IS) (Gabbay and Rodrigues 2015) & $\begin{array}{l}\operatorname{Deg}_{\mathrm{G}}^{\mathrm{IS}}(a)=\lim _{i \rightarrow+\infty} f_{i}(a), \text { where } \\
f_{i}(a)=\left(1-f_{i-1}(a)\right) \min \left(\frac{1}{2}, 1-\max _{b \mathcal{R} a} f_{\mathrm{i}-1}(\mathrm{~b})\right)+ \\
f_{i-1}(a) \max \left(\frac{1}{2}, 1-\max _{b \mathcal{R} a} f_{\mathrm{i}-1}(\mathrm{~b})\right)\end{array}$ & Weighted \\
\hline
\end{tabular}

Table 1: Gradual semantics dealing with cycles.

Definition 9. Let $\mathbf{S} \in\{i, s, p, g\}, \mathrm{G}=\langle\mathcal{A}, \sigma \equiv 1, \mathcal{R}\rangle \in \mathrm{AG}$ and $a \in \mathcal{A}$. If $\mathrm{G}$ has no extensions, then $\operatorname{Deg}_{\mathrm{G}}^{\mathbf{S}}(a)=\beta$. Otherwise,

- $\operatorname{Deg}_{G}^{\mathbf{S}}(a)=1$ iff a belongs to all extensions.

- $\operatorname{Deg}_{\mathrm{G}}^{\mathbf{S}}(a)=\alpha$ iff a is in some but not all extensions.

- $\operatorname{Deg}_{G}^{\mathbf{S}}(a)=\beta$ iff a does not belong to any extension and is not attacked by any extension.

- $\operatorname{Deg}_{G}^{\mathbf{S}}(a)=0$ iff a does not belong to any extension and is attacked by at least one extension.

Depending on the semantics some values of the scale $T$ may not be used. Indeed, under grounded and ideal, an argument can never get the value $\alpha$. When stable extensions exist, an argument cannot receive the value $\beta$.

Property 6. Let $\mathrm{G}=\langle\mathcal{A}, \sigma \equiv 1, \mathcal{R}\rangle \in \mathrm{AG}$ and $a \in \mathcal{A}$.

- $\operatorname{Deg}_{G}^{g}(a) \in T \backslash\{\alpha\}$

- $\operatorname{Deg}_{G}^{i}(a) \in T \backslash\{\alpha\}$

- If stable extensions exist, $\operatorname{Deg}_{G}^{s}(a) \in T \backslash\{\beta\}$.

From their definitions, the four semantics seem compatible. For instance, grounded is more sceptical than ideal, which in turn is more sceptical than preferred and stable. The following result confirms this observation, however it shows that the four semantics are only weakly equivalent. None of them refines or is strongly equivalent to the others.

Theorem 1. The four semantics (stable, preferred, grounded, ideal) are pairwise weakly equivalent.

Consider the following example.

Example 1. Consider the following flat argumentation graph $\mathrm{G}$, and let us focus on grounded and stable semantics.

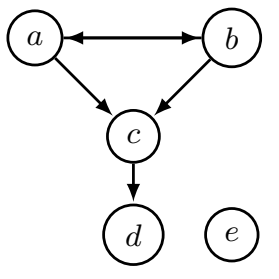

\section{Grounded semantics:}

- $\operatorname{Gr}(\mathrm{G})=\{e\}$

- $\operatorname{Deg}_{\mathrm{G}}^{g}(e)=1$ and $\operatorname{Deg}_{\mathrm{G}}^{g}(a)=\operatorname{Deg}_{\mathrm{G}}^{g}(b)=\operatorname{Deg}_{\mathrm{G}}^{g}(c)=$ $\operatorname{Deg}_{\mathrm{G}}^{g}(d)=\beta$.

- $e \succ_{g} a \approx_{g} b \approx_{g} c \approx_{g} d$

Stable semantics:

- The graph has two extensions $\{a, d, e\}$ and $\{b, d, e\}$.

- $\operatorname{Deg}_{G}^{s}(d)=\operatorname{Deg}_{G}^{s}(e)=1, \operatorname{Deg}_{G}^{s}(a)=\operatorname{Deg}_{G}^{s}(b)=\alpha$ and $\operatorname{Deg}_{\mathrm{G}}^{s}(c)=0$.

- $d \approx_{s} e \succ_{s} a \approx_{s} b \succ_{s} c$

Note that the stable semantics does not refine the grounded since $\succ_{g} \nsubseteq \succ_{s}$. The two semantics are also not strongly equivalent since $\succeq_{g} \neq \succeq_{s}$.

\section{Comparison of Gradual Semantics}

Initiated in (Cayrol and Lagasquie-Schiex 2005), gradual semantics have received increasing interest during the last few years due to their fine-grained evaluations of arguments. Indeed, several semantics have been proposed in the literature, some of which deal only with acyclic graphs like QuAD (Baroni et al. 2015) and DFQuAD (Rago et al. 2016) and others are suitable for any graph typology. Since we are interested by comparison of 
pairs of semantics, we focus in this paper on the second type of semantics and more precisely on the eight recalled in Table 1 . Note that the two first semantics (hCat and $\alpha-\mathrm{BBS}$ ) deal only with flat graphs. In what follows, we present results that hold when argumentation graphs are weighted and others that hold in case of flat graphs.

The first result states that hCat and $\alpha-\mathrm{BBS}$ are strongly equivalent. Furthermore, in case of flat graphs (i.e. graphs with $\sigma \equiv 1$ ), hCat and Weighted $h$-categorizer (Hbs) are also strongly equivalent since they coincide. These three semantics are fully similar since they provide both the same evaluations of individual arguments and the same rankings.

Theorem 2. In case of flat graphs, hCat, $\alpha-\mathrm{BBS}$ (with $\alpha=$ 1) and $\mathrm{Hbs}$ are pairwise strongly equivalent.

The six semantics that deal with weighted graphs are pairwise incompatible, that is, they may return contradictory rankings for pairs of arguments. They are thus all different, and the choice of the one to use depends on the application and the nature of arguments (deductive, analogical, ...).

Theorem 3. The six semantics $\mathrm{Hbs}, \mathrm{Mbs}, \mathrm{Cbs}, \mathrm{EMbs}, \mathrm{TB}$ and IS are pairwise incompatible.

It is worthy to recall that Mbs and EMbs satisfy exactly the same subset of principles (see (Amgoud and Doder 2019)). However, they may disagree on their ranking of arguments as shown in the following example.

Example 2. Consider the weighted graph below where $\sigma(a)=0.6252, \sigma(b)=0.3939$ and $\sigma(c)=0.8107$.

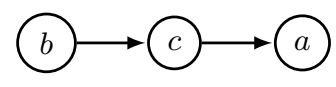

It can be checked that $\operatorname{Deg}_{G}^{\mathrm{Mbs}}(a)=0.3953, \operatorname{Deg}_{\mathrm{G}}^{\mathrm{Mbs}}(b)=$ $0.3939, \operatorname{Deg}_{G}^{\mathrm{EMbs}}(a)=0.362$, and $\operatorname{Deg}_{G}^{\mathrm{EMbs}}(b)=0.3939$. Note that $a \succ_{\mathrm{Mbs}} b$ and $b \succ_{\mathrm{EMbs}} a$. This shows that the two semantics may lead to different results.

Let us now focus only on flat argumentation graphs (i.e. graphs of the form $\mathrm{G}=\langle\mathcal{A}, \sigma \equiv 1, \mathcal{R}\rangle$ ), and let us investigate the relations between the four gradual semantics (Mbs, EMbs, TB, IS). These all use the same function, namely max, for aggregating the strengths of an argument's attackers. In other words, they consider only the strongest attacker when computing the strength of any argument.

We show that the two semantics (TB, IS) coincide, i.e. they assign exactly the same value to an argument. Recall that this property is not true in the general case of weighted graphs. We show also that the value of an argument depends on whether the argument is in or is attacked by the grounded extension $\operatorname{Gr}(\mathrm{G})$ of the graph. This means that (TB, IS) are two alternative characterizations of the grounded semantics.

Theorem 4. For any $\mathrm{G}=\langle\mathcal{A}, \sigma \equiv 1, \mathcal{R}\rangle \in \mathrm{AG}$, it holds that

$$
\operatorname{Deg}_{G}^{\mathrm{TB}} \equiv \operatorname{Deg}_{G}^{\mathrm{IS}} .
$$

Moreover, for any $x \in\{\mathrm{TB}, \mathrm{IS}\}$, for any $a \in \mathcal{A}$,

- $\operatorname{Deg}_{\mathrm{G}}^{x}(a)=1$ iff $a \in \operatorname{Gr}(\mathrm{G})$.

- $\operatorname{Deg}_{G}^{x}(a)=0$ iff $\operatorname{Gr}(G)$ attacks $a$.
- $\operatorname{Deg}_{\mathrm{G}}^{x}(a)=\frac{1}{2}$ iff $a \notin \mathrm{Gr}(\mathrm{G})$ and $\operatorname{Gr}(\mathrm{G})$ does not attack $a$.

The two semantics (TB, IS) are thus strongly equivalent when dealing with flat graphs.

Theorem 5. In case of flat argumentation graphs, TB and IS are strongly equivalent.

The two semantics Mbs and EMbs also are strongly equivalent when applied to flat graphs. Before presenting the formal result, we start by providing novel characterizations of Mbs and EMbs using two well-known sequences: Fibonacci for Mbs and Exponential for EMbs.

When a graph $\mathrm{G}$ is flat, the strength of an argument, say $a$, under Mbs is given by the following equation:

$$
\operatorname{Deg}_{G}^{M b s}(a)=\frac{1}{1+m_{b \mathcal{R} a} a \operatorname{Deg}_{G}^{M b s}(b)} .
$$

It has been shown in (Amgoud et al. 2017) that $\operatorname{Deg}_{G}^{M b s}(a) \in$ $\left[\frac{1}{2}, 1\right]$. In what follows, we show that the values assigned to arguments by Mbs are not arbitrary, they are rather the Fibonacci numbers, i.e. elements of the Fibonacci sequence $\left\{F^{n}\right\}_{n \geq 0}$ defined as follows:

$$
F^{0}=0 \quad F^{1}=1 \quad F^{n}=F^{n-1}+F^{n-2} \text { for } n>1 .
$$

We get the sequence of so-called Fibonacci numbers: $\langle 0,1,1,2,3,5,8,13,21,34,55,89,144,233,377,610, \ldots\rangle$. It has been shown in (Philippou 2015) that the ratio of two consecutive Fibonacci numbers (i.e. $\frac{F^{n}}{F^{n-1}}$ ) tends to the so-called golden ratio $\varphi=\frac{1+\sqrt{5}}{2}$ as $n$ increases.

From the Fibonacci sequence $\left\{F^{n}\right\}_{n \geq 0}$, we define another sequence $\left\{\mathcal{S}^{n}\right\}_{n \geq 1}$ such that:

$$
\mathcal{S}^{n}=\frac{F^{n}}{F^{n+1}}
$$

Let $\mathcal{S}=\left\{1, \frac{1}{2}, \frac{2}{3}, \frac{3}{5}, \frac{5}{8}, \frac{8}{13}, \frac{13}{21}, \frac{21}{34}, \frac{34}{55}, \frac{55}{89}, \frac{89}{144}, \frac{144}{233}, \frac{233}{377}, \ldots\right\}$ be the set of all numbers of $\left\{\mathcal{S}^{n}\right\}_{n \geq 1}$. It is worth noticing that this sequence contains two sub-sequences: The decreasing sub-sequence

$$
\mathcal{S}_{1}=\left\langle 1, \frac{2}{3}, \frac{5}{8}, \frac{13}{21}, \frac{34}{55}, \frac{89}{144}, \frac{233}{377}, \ldots\right\rangle
$$

made of the numbers that are at odd positions in $\mathcal{S}$, and the increasing sub-sequence

$$
\mathcal{S}_{2}=\left\langle\frac{1}{2}, \frac{3}{5}, \frac{8}{13}, \frac{21}{34}, \frac{55}{89}, \frac{144}{233}, \ldots\right\rangle
$$

which contains the numbers that are at even positions in $\mathcal{S}$.

Notation: We denote by $\mathcal{S}_{x}^{i}$ the $i^{\text {th }}$ element of the subsequence $\mathcal{S}_{x}$ where $x \in\{1,2\}$. For instance, $\mathcal{S}_{1}^{1}=1$, $\mathcal{S}_{2}^{1}=\frac{1}{2}$ and $\mathcal{S}_{2}^{3}=\frac{8}{13}$.

It is worthy to recall that the two sub-sequences converge, furthermore they have the same limit. Indeed,

$$
\lim _{n \rightarrow \infty} \mathcal{S}_{1}^{n}=\lim _{n \rightarrow \infty} \mathcal{S}_{2}^{n}=\frac{1}{\varphi} .
$$


It is also well-known that

$$
\mathcal{S}_{2}^{n}<\frac{1}{\varphi}<\mathcal{S}_{1}^{n}, \quad \forall n \geq 1 .
$$

In case of a flat graph G, the semantics Mbs takes its values from the sequence $\mathcal{S}$, i.e. $\operatorname{Deg}_{G}^{\mathrm{Mbs}}(.) \in \mathcal{S}$. It uses the above three parts of the sequence $\mathcal{S}$, namely $\mathcal{S}_{1}, \mathcal{S}_{2}, \frac{1}{\varphi}$, for distinguishing between three groups of arguments in $\mathrm{G}$ :

- The first group is composed of all elements of the set $\bigcup_{i>1} \mathcal{F}^{i}(\emptyset)$, with $\mathcal{F}$ being the characteristic function defined by (Dung 1995) and that returns all the arguments defended by a given set of arguments. Hence, this group contains all arguments that are defended (directly or indirectly) by non attacked arguments in G. Its members are stronger than any argument outside the group, and their values are taken from the sub-sequence $\mathcal{S}_{1}$.

- The second group contains the arguments that are attacked by at least one argument of the set $\bigcup_{i \geq 1} \mathcal{F}^{i}(\emptyset)$. These will be weaker than any argument of the two other groups, and their values are numbers of the sub-sequence $\mathcal{S}_{2}$.

- The third group contains all the remaining arguments, i.e. those that are neither in nor attacked by the set $\bigcup_{i \geq 1} \mathcal{F}^{i}(\emptyset)$. The semantics Mbs ascribes the value $\frac{1}{\varphi}$ to every argument of this group.

Unlike the third group, the semantics may assign different values to arguments of the first group. It is interesting to note that the value of an argument depends on the iteration at which it appears for the first time in the set $\bigcup_{i \geq 1} \mathcal{F}^{i}(\emptyset)$. Recall that $\bigcup_{i \geq 1} \mathcal{F}^{i}(\emptyset)=\mathcal{F}^{1}(\emptyset) \cup \mathcal{F}^{2}(\emptyset) \cup \ldots \mathcal{F}^{n}(\emptyset)$, with $\mathcal{F}^{i}(\emptyset)=$ $\mathcal{F}\left(\mathcal{F}^{i-1}(\emptyset)\right)$ is the set of arguments defended by $\mathcal{F}^{i-1}(\emptyset)$. If an argument $a$ appears for the first time in $\mathcal{F}^{i}(\emptyset)$, then it gets the value of the $i^{t h}$ element of $\mathcal{S}_{1}$, namely $\mathcal{S}_{1}^{i}$. Furthermore, every argument it attacks receive the value $\mathcal{S}_{2}^{i}$ meaning that arguments of the second group do not necessarily have the same strength as well.

Theorem 6. Let $\mathrm{G}=\langle\mathcal{A}, \sigma \equiv 1, \mathcal{R}\rangle \in \mathrm{AG}$ and $i \in \mathbb{N}$. For any $a \in \mathcal{A}$, $\operatorname{Deg}_{G}^{\mathrm{Mbs}}(a) \in \mathcal{S}$. Furthermore,

$$
\begin{aligned}
& \text { - } \operatorname{Deg}_{G}^{M b s}(a)=\mathcal{S}_{1}^{i} \text { iff } a \in \mathcal{F}^{i}(\emptyset) \text { and } a \notin \bigcup_{j=1}^{i-1} \mathcal{F}^{j}(\emptyset) . \\
& \text { - } \operatorname{Deg}_{G}^{\text {Mbs }}(a)=\mathcal{S}_{2}^{i} \text { iff } \mathcal{F}^{i}(\emptyset) \text { attacks } a \text { and } \bigcup_{j=1}^{i-1} \mathcal{F}^{j}(\emptyset) \text { does } \\
& \text { not attack a. } \\
& \text { - } \operatorname{Deg}_{G}^{\text {Mbs }}(a)=\frac{1}{\varphi} \text { iff } a \notin \bigcup \mathcal{F}^{i \geq 1}(\emptyset) \text { and } \bigcup \mathcal{F}^{i \geq 1}(\emptyset) \text { does } \\
& \\
& \text { not attack a. }
\end{aligned}
$$

Note that according to Mbs, the longer the defence path of an argument, the weaker the argument.

Example 3. Consider the flat graph depicted below: Note that $\mathcal{F}^{1}(\emptyset)=\{a\}, \mathcal{F}^{2}(\emptyset)=\{a, d\}$ and $\mathcal{F}^{3}(\emptyset)=$ $\{a, d, f\}$. It can be checked that: $\operatorname{Deg}^{\text {Mbs }}(a)=\mathcal{S}_{1}^{1}=1$, $\operatorname{Deg}^{\mathrm{Mbs}}(d)=\mathcal{S}_{1}^{2}=\frac{2}{3}$, and $\operatorname{Deg}{ }^{\mathrm{Mbs}}(f)=\mathcal{S}_{1}^{3}=\frac{5}{8}$. Since

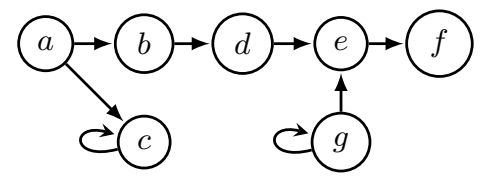

$\mathcal{F}^{1}(\emptyset)$ attacks both $b, c$, then $\operatorname{Deg}^{\mathrm{Mbs}}(b)=\operatorname{Deg}^{\mathrm{Mbs}}(c)=$ $\mathcal{S}_{2}^{1}=\frac{1}{2}$, and since $\mathcal{F}^{2}(\emptyset)$ attacks $e$, then $\operatorname{Deg}^{\mathrm{Mbs}}(e)=\mathcal{S}_{2}^{2}=$ $\frac{3}{5}$. Finally, $\operatorname{Deg}^{\mathrm{Mbs}}(g)=\frac{1}{\varphi}$.

Let us now provide a novel characterization of the Euler Max-based (EMbs) semantics in case of a flat graph G. Recall that the strength of an argument $a$ is computed as follows:

$$
\operatorname{Deg}_{G}^{\mathrm{EMbs}}(a)=e^{-\max _{b \mathcal{R} a} \operatorname{Deg}_{G}^{\mathrm{EMbs}}(b)} .
$$

We show that EMbs takes its values from the set of numbers of the following exponential sequence $\left\{\mathcal{U}^{n}\right\}_{n \geq 0}$ such that:

$$
\mathcal{U}^{1}=1 \quad \mathcal{U}^{n}=e^{-\mathcal{U}^{n-1}} \text { for } n>1
$$

Let $\mathcal{U}=\{1,0.3678,0.6922,0.5004,0.6062,0.5453$, $0.5796, \ldots\}$. Like the Fibonacci sequence, $\left\{\mathcal{U}^{n}\right\}_{n \geq 0}$ contains two sub-sequences: The decreasing sub-sequence

$$
\mathcal{U}_{1}=\langle 1,0.6922,0.6062,0.5796, \ldots\rangle
$$

made of the numbers that are at odd positions in $\mathcal{U}$, and the increasing sub-sequence

$$
\mathcal{U}_{2}=\langle 0.3678,0.5004,0.5453, \ldots\rangle
$$

which contains the numbers that are at even positions in $\mathcal{U}$. We denote by $\mathcal{U}_{x}^{i}$ the $i^{\text {th }}$ element of the sub-sequence $\mathcal{U}_{x}$ where $x \in\{1,2\}$. Finally, it is well-known that the two sub-sequences converge to the same limit $\Omega$,

$$
\lim _{n \rightarrow \infty} \mathcal{U}_{1}^{n}=\lim _{n \rightarrow \infty} \mathcal{U}_{2}^{n}=\Omega
$$

where $\Omega \approx 0.5671432904$ is the so-called Omega Constant and

$$
\mathcal{U}_{2}^{n}<\Omega<\mathcal{U}_{1}^{n}, \quad \forall n \geq 1 .
$$

Like Mbs, the semantics EMbs divides the set of arguments into three groups: i) those that belong to the set $\bigcup_{i \geq 1} \mathcal{F}^{i}(\emptyset)$, ii) those attacked by $\bigcup_{i \geq 1} \mathcal{F}^{i}(\emptyset)$, and iii) the remaining ones.

Theorem 7. Let $\mathrm{G}=\langle\mathcal{A}, \sigma \equiv 1, \mathcal{R}\rangle \in \mathrm{AG}$ and $i \in \mathbb{N}$. For any $a \in \mathcal{A}$, $\operatorname{Deg}^{\mathrm{EMbs}}(a) \in \mathcal{U}$. Furthermore,

- $\operatorname{Deg}_{G}^{\text {EMbs }}(a)=\mathcal{U}_{1}^{i}$ iff $a \in \mathcal{F}^{i}(\emptyset)$ and $a \notin \bigcup_{j=1}^{i-1} \mathcal{F}^{j}(\emptyset)$.

- $\operatorname{Deg}_{G}^{\mathrm{EMbs}}(a)=\mathcal{U}_{2}^{i}$ iff $\mathcal{F}^{i}(\emptyset)$ attacks $a$ and $\bigcup_{j=1}^{i-1} \mathcal{F}^{j}(\emptyset)$ does not attack $a$.

- $\operatorname{Deg}_{G}^{\text {EMbs }}(a)=\Omega$ iff $a \notin \bigcup \mathcal{F}^{i \geq 1}(\emptyset)$ and $\bigcup \mathcal{F}^{i \geq 1}(\emptyset)$ does not attack $a$.

Example 3 (Cont) It can be checked that $\operatorname{Deg}^{\mathrm{EMbs}}(a)=$ $\mathcal{U}_{1}^{1}=1, \operatorname{Deg}^{\mathrm{EMbs}}(d)=\mathcal{U}_{1}^{2}=0.6922$, and $\operatorname{Deg}^{\mathrm{EMbs}}(f)=$ $\mathcal{U}_{1}^{3}=0.6062$. Since $\mathcal{F}^{1}(\emptyset)$ attacks both $b, c$, then $\operatorname{Deg}^{\mathrm{EMbs}}(b)=\operatorname{Deg}^{\mathrm{EMbs}}(c)=\mathcal{U}_{2}^{1}=0.3678$, and since $\mathcal{F}^{2}(\emptyset)$ attacks $e$, then $\operatorname{Deg}^{\mathrm{EMbs}}(e)=\mathcal{U}_{2}^{2}=0.5004$. Finally, $\operatorname{Deg}^{\mathrm{EMbs}}(g)=\Omega$. 
Notations: Let $\mathrm{G}$ be a flat graph, $A, B$ be two sets of arguments and $x \in$ Sem. The notation $A \succ_{x} B$ stands for $\forall a \in A, \forall b \in B$, it holds $\operatorname{Deg}^{x}(a)>\operatorname{Deg}^{x}(b)$. Let $A^{+}$denote the set of arguments that are attacked by at least one argument of $A$, and $A^{\circ}$ be the set of arguments that are neither in nor attacked by the set $A$. Let $\mathcal{X}_{i}=\mathcal{F}^{i}(\emptyset) \backslash\left(\bigcup_{j=1}^{i-1} \mathcal{F}^{j}(\emptyset)\right)$ with $i>1$ and by convention, $\mathcal{X}_{1}=\mathcal{F}^{1}(\emptyset)$. This means that $\mathcal{X}_{1}=\mathcal{F}(\emptyset), \mathcal{X}_{2}=\mathcal{F}^{2}(\emptyset) \backslash \mathcal{F}^{1}(\emptyset)$, and so on.

The following result follows from the characterizations of Mbs, EMbs. It shows how the two semantics refine the grounded extension of a graph, and how they refine the set of arguments attacked by the grounded extension.

Theorem 8. Let $\mathrm{G}$ be a flat graph and $x \in\{\mathrm{Mbs}, \mathrm{EMbs}\}$.

- $\mathcal{X}_{1} \succ_{x} \mathcal{X}_{2} \succ_{x} \ldots \succ_{x} \mathcal{X}_{n}$.

- $\mathcal{X}_{n}^{+} \succ_{x} \mathcal{X}_{n-1}^{+} \succ_{x} \ldots \succ_{x} \mathcal{X}_{1}^{+}$.

- $\operatorname{Gr}(\mathrm{G}) \succ_{x} \operatorname{Gr}(\mathrm{G})^{o} \succ_{x} \operatorname{Gr}(\mathrm{G})^{+}$.

From the characterizations of Mbs and EMbs, it follows that the two semantics are strongly equivalent.

Theorem 9. In case of flat argumentation graphs, Mbs and EMbs are strongly equivalent.

The previous results show that TB and IS assign the same value to any argument that belongs to (or is attacked by) the grounded extension. Mbs and EMbs are more discriminating and provide finer-grained evaluations and comparisons of arguments. Thus, they both refine TB and IS.

Theorem 10. In case of flat argumentation graphs, Mbs (resp. EMbs) refines TB (resp. IS).

The novel characterizations of the four semantics Mbs, EMbs, TB and IS show that they are based on the grounded extension of a graph $\mathrm{G}$ as $\operatorname{Gr}(\mathrm{G})=\bigcup_{i \geq 1} \mathcal{F}^{i}(\emptyset)$. They can also be seen as different characterizations of the grounded semantics in terms of various gradual semantics.

\section{Extension Semantics vs Gradual Semantics}

Throughout this section, we focus on flat argumentation graphs. We show that hCat, $\alpha-\mathrm{BBS}, \mathrm{Hbs}$ and $\mathrm{Cbs}$ are incompatible with any of the recalled extension semantics. This is mainly due to the fact that the first group takes into account all the attackers of an argument while the second focuses only on the strongest one.

Theorem 11. hCat (resp. $\alpha-\mathrm{BBS}, \mathrm{Hbs}$, Cbs) is incompatible with grounded, ideal, stable and preferred.

Unsurprisingly, the two semantics TB and IS are strongly equivalent with the grounded semantics. Furthermore, they are weakly equivalent to the three other extension semantics.

Theorem 12. In case of flat argumentation graphs, the following properties hold:

- TB, IS and grounded are pairwise strongly equivalent.

- TB (resp. IS) is weakly equivalent with ideal (resp. stable and preferred).
We show also that Mbs (resp. EMbs) refines the grounded semantics when applied to flat argumentation graphs. However, the converse does not hold, thus the two semantics are not strongly equivalent (see Example 3).

Theorem 13. In case of flat argumentation graphs, Mbs (resp. EMbs) refines the grounded semantics.

Mbs (resp. EMbs) does not refine ideal, stable and preferred semantics. It is thus not strongly equivalent with any of these three semantics as shown below.

Example 4. Consider the flat graph below. It has a single preferred (respectively stable) extension which coincides with the ideal and grounded extension $\{a, c\}$. Thus, $a \equiv_{x} c$, with $x \in\{i, s, p\}$, while $a \succ_{\mathrm{Mbs}} c$ and $a \succ_{\mathrm{EMbs}} c$.

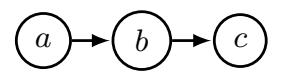

The last result shows that the Mbs (resp. EMbs) semantics is weakly equivalent with ideal, stable and Preferred semantics. They provide thus compatible rankings of arguments.

Theorem 14. In case of flat argumentation graphs, Mbs (resp. EMbs) is weakly equivalent with ideal, stable and preferred semantics.

Table 2 summarizes the relations between the reviewed semantics in case of flat argumentation graphs. There are 5 groups of semantics. The first group contains (hCat, Hbs, $\alpha-$ BBS), which are strongly equivalent, provide the same evaluations for individual arguments, and are incompatible with all the other semantics. The second group contains only $\mathrm{Cbs}$, which is incompatible with any other semantics. Like the semantics of the first group, with Cbs every attacker is harmful to its target. However, Cbs favors the quantity of attackers while the former promote compensation. The third group is made of Mbs, EMbs, which are strongly equivalent, and refine some semantics that consider only one attacker, namely TB, IS and grounded. The fourth group is made of these three semantics, which are strongly equivalent. The last group contains ideal, stable and preferred, which are incompatible with semantics of the first and second groups, and are weakly equivalent with any of the remaining semantics.

An important question is which semantics to choose in a given application? In (Amgoud and Ben-Naim 2013; Rago, Baroni, and Toni 2018), the authors have argued that the choice of the suitable semantics depends on the nature of arguments (deductive, analogical, causal, ...) and the specificities of the application. It is worth mentioning that existing principles provide a good basis for assessing the suitability of a semantics. However, they are insufficient since two semantics may satisfy the same principles and still return opposite rankings. This means the two semantics may lead to different outcomes in decision making, for instance.

\section{Related Work}

As said in the introduction, several works have been devoted to the comparison of semantics. They focused on the definition of principles and analysis of semantics against them. 


\begin{tabular}{|l||c|c|c|c|c|c|c|c|c|c|c|c|}
\hline Semantics & hCat & $\alpha$-BBS & Hbs & Cbs & Mbs & EMbs & TB & IS & Grounded & Ideal & Stable & Preferred \\
\hline \hline hCat & - & $s$ & $s$ & $i$ & $i$ & $i$ & $i$ & $i$ & $i$ & $i$ & $i$ & $i$ \\
\hline$\alpha-$ BBS & $s$ & - & $s$ & $i$ & $i$ & $i$ & $i$ & $i$ & $i$ & $i$ & $i$ & $i$ \\
\hline Hbs & $s$ & $s$ & - & $i$ & $i$ & $i$ & $i$ & $i$ & $i$ & $i$ & $i$ & $i$ \\
\hline \hline Cbs & $i$ & $i$ & $i$ & - & $i$ & $i$ & $i$ & $i$ & $i$ & $i$ & $i$ & $i$ \\
\hline \hline Mbs & $i$ & $i$ & $i$ & $i$ & - & $s$ & $r$ & $r$ & $r$ & $w$ & $w$ & $w$ \\
\hline EMbs & $i$ & $i$ & $i$ & $i$ & $s$ & - & $r$ & $r$ & $r$ & $w$ & $w$ & $w$ \\
\hline \hline TB & $i$ & $i$ & $i$ & $i$ & $w$ & $w$ & - & $s$ & $s$ & $w$ & $w$ & $w$ \\
\hline IS & $i$ & $i$ & $i$ & $i$ & $w$ & $w$ & $s$ & - & $s$ & $w$ & $w$ & $w$ \\
\hline Grounded & $i$ & $i$ & $i$ & $i$ & $w$ & $w$ & $s$ & $s$ & - & $w$ & $w$ & $w$ \\
\hline \hline Ideal & $i$ & $i$ & $i$ & $i$ & $w$ & $w$ & $w$ & $w$ & $w$ & - & $w$ & $w$ \\
\hline Stable & $i$ & $i$ & $i$ & $i$ & $w$ & $w$ & $w$ & $w$ & $w$ & $w$ & - & $w$ \\
\hline Preferred & $i$ & $i$ & $i$ & $i$ & $w$ & $w$ & $w$ & $w$ & $w$ & $w$ & $w$ & - \\
\hline
\end{tabular}

Table 2: Case of Flat Argumentation Graphs. $s, w, r, i$ stand resp. for strong equivalence, weak equivalence, refinement, incompatibility.

To the best of our knowledge, this is the first work that compares semantics wrt their rankings.

An important question is how this work relates to ranking semantics that have been introduced by (Amgoud and Ben-Naim 2013). Those semantics take as input a flat argumentation graph, and rank arguments according to their strengths. Some existing ranking semantics have been compared by (Bonzon et al. 2016) on the basis of the principles they satisfy. However, as for gradual semantics, the fact that two ranking semantics satisfy the same principles does not necessarily mean they provide the same ranking. The principles ensure only some rational behaviour of semantics. Finally, it is worth mentioning that (Baumann et al. 2019) studied a different notion of equivalence in argumentation. While we focused on equivalence of semantics, they were interested in comparing argumentation graphs. They check the extensions (under various extensionbased semantics) they deliver under any addition of arguments and/or attacks.

\section{Conclusion}

The paper has tackled the question of comparing semantics, be them extension-based or gradual. It has shown that existing comparisons in the literature are not conclusive, since they were only based on the evaluations of individual arguments. It has argued that comparing rankings is also crucial for the similarity of semantics. The paper has thus investigated a notion of equivalence which is based on the comparison of rankings, and has studied several existing semantics. The results have shown compatibilities between the classical extension semantics and the gradual semantics that consider only the stronger attacker of an argument when computing its strength. Some gradual semantics like TB, IS go further by providing characterizations for the grounded semantics, while Mbs, EMbs refine the latter in a reasoned way. The gradual semantics that consider all attackers are incompatible with extension semantics. Furthermore, they are themselves pairwise incompatible in case of weighted graphs.

This work will be extended in different directions. First, we plan to compare extension semantics that take into ac- count preferences with gradual semantics. We will also look for novel principles that can distinguish between Mbs and EMbs in case of weighted graphs. Indeed, the two semantics satisfy exactly the same subset of existing principles but may provide opposite rankings when graphs are weighted.

\section{Acknowledgements}

Support from the ANR-3IA Artificial and Natural Intelligence Toulouse Institute is gratefully acknowledged.

\section{References}

Amgoud, L., and Ben-Naim, J. 2013. Ranking-based semantics for argumentation frameworks. In Proceedings of the 7th International Conference Scalable on Uncertainty Management SUM, 134-147.

Amgoud, L., and Ben-Naim, J. 2016. Axiomatic foundations of acceptability semantics. In Proceedings of the fifteenth International Conference on Principles of Knowledge Representation and Reasoning KR, 2-11.

Amgoud, L., and Cayrol, C. 2002. Inferring from inconsistency in preference-based argumentation frameworks. International Journal of Automated Reasoning 29(2):125-169.

Amgoud, L., and Doder, D. 2019. Gradual semantics accounting for varied-strength attacks. In Proceedings of the 18th International Conference on Autonomous Agents and MultiAgent Systems, AAMAS, 1270-1278.

Amgoud, L., and Prade, H. 2009. Using arguments for making and explaining decisions. Artificial Intelligence 173:413-436.

Amgoud, L.; Ben-Naim, J.; Doder, D.; and Vesic, S. 2016. Ranking arguments with compensation-based semantics. In Proceedings of the fifteenth International Conference on Principles of Knowledge Representation and Reasoning KR, $12-21$.

Amgoud, L.; Ben-Naim, J.; Doder, D.; and Vesic, S. 2017. Acceptability semantics for weighted argumentation frameworks. In Proceedings of the International Joint Conference on Artificial Intelligence IJCAI, 56-62. 
Baroni, P., and Giacomin, M. 2007. On principle-based evaluation of extension-based argumentation semantics. Artificial Intelligence 171(10-15):675-700.

Baroni, P.; Romano, M.; Toni, F.; Aurisicchio, M.; and Bertanza, G. 2015. Automatic evaluation of design alternatives with quantitative argumentation. Argument \& Computation 6(1):24-49.

Baroni, P.; Giacomin, M.; and Guida, G. 2005. Sccrecursiveness: a general schema for argumentation semantics. Artif. Intell. 168(1-2):162-210.

Baumann, R.; Dvorák, W.; Linsbichler, T.; and Woltran, S. 2019. A general notion of equivalence for abstract argumentation. Artificial Intelligence 275:379-410.

Benferhat, S.; Dubois, D.; and Prade, H. 1993. Argumentative inference in uncertain and inconsistent knowledge bases. In International Conference on Uncertainty in AI UAI, 411-419.

Besnard, P., and Hunter, A. 2001. A logic-based theory of deductive arguments. Artificial Intelligence 128(1-2):203235.

Bonzon, E.; Delobelle, J.; Konieczny, S.; and Maudet, N. 2016. A comparative study of ranking-based semantics for abstract argumentation. In Proceedings of the AAAI Conference on Artificial Intelligence (AAAI), 914-920.

Cayrol, C., and Lagasquie-Schiex, M. 2005. Graduality in argumentation. Journal of Artificial Intelligence Research 23:245-297.

Cyras, K.; Satoh, K.; and Toni, F. 2016. Explanation for case-based reasoning via abstract argumentation. In Proceedings of Computational Models of Argument, volume 287, 243-254.

da Costa Pereira, C.; Tettamanzi, A.; and Villata, S. 2011. Changing one's mind: Erase or rewind? In Proceedings of the International Joint Conference on Artificial Intelligence IJCAI, 164-171.

Dimopoulos, Y.; Mailly, J.; and Moraitis, P. 2019. Argumentation-based negotiation with incomplete opponent profiles. In Proceedings of the 18th International Conference on Autonomous Agents and MultiAgent Systems, 12521260.

Dung, P.; Mancarella, P.; and Toni, F. 2007. Computing ideal skeptical argumentation. Artificial Intelligence 171:642-674.

Dung, P. M. 1995. On the Acceptability of Arguments and its Fundamental Role in Non-Monotonic Reasoning, Logic Programming and n-Person Games. Artificial Intelligence 77:321-357.

Gabbay, D. M., and Rodrigues, O. 2015. Equilibrium states in numerical argumentation networks. Logica Universalis 9(4):411-473.

Grossi, D., and Modgil, S. 2015. On the graded acceptability of arguments. In Proceedings of the 24th International Joint Conference on Artificial Intelligence, IJCAI'15, 868-874.

Leite, J., and Martins, J. 2011. Social abstract argumentation. In Proceedings of the International Joint Conference on Artificial Intelligence IJCAI, 2287-2292.
Philippou, A. 2015. Fibonacci numberts, probability and gambling. In Proceedings of the International Conference on Mathematics Education and Mathematics in Engineering and Technology, (ICMET), 13-21.

Rago, A.; Baroni, P.; and Toni, F. 2018. On instantiating generalised properties of gradual argumentation frameworks. In 12th International Conference on Scalable Uncertainty Management, SUM, volume 11142 of Lecture Notes in Computer Science, 243-259.

Rago, A.; Toni, F.; Aurisicchio, M.; and Baroni, P. 2016. Discontinuity-free decision support with quantitative argumentation debates. In Proceedings of the fifteenth International Conference on Principles of Knowledge Representation and Reasoning $K R, 63-73$.

Simari, G. R., and Rahwan, I., eds. 2009. Argumentation in Artificial Intelligence. Springer.

van der Torre, L., and Vesic, S. 2017. The principle-based approach to abstract argumentation semantics. FLAP 4(8).

Zheng, H.; Grossi, D.; and Verheij, B. 2020. Case-based reasoning with precedent models: Preliminary report. In Proceedings of Computational Models of Argument, volume 326, 443-450.

Zhong, Q.; Fan, X.; Luo, X.; and Toni, F. 2019. An explainable multi-attribute decision model based on argumentation. Expert Systems with Applications 117:42-61. 